\title{
STABILITY ANALYSIS OF A COMPLIANT LEMON BORE JOURNAL BEARING
}

\author{
Evgeny Kuznetsov \\ Division of Machine Elements \\ Luleå University of Technology \\ 97187 Luleå, Sweden \\ evgeny@ltu.se
}

\author{
Sergei Glavatskih \\ Division of Machine Elements \\ Luleå University of Technology \\ 97187 Luleå, Sweden \\ segla@ltu.se
}

\begin{abstract}
Dynamic coefficients for a lemon bore journal bearing with a compliant PTFE surface are computed. An efficient method for the calculation of bearing dynamic coefficients proposed by Lund [4] has been extended to compliant bearings. Comparison with a rigid babbitted lemon bore journal bearing in terms of dynamic coefficients and stability is given and discussed. It is shown that it is important to consider perturbations of both the oil film and the compliant lining to obtain realistic results. It is also shown that stability of compliant bearings is improved at some eccentricities compared with conventional babbitted bearings.
\end{abstract}

\section{Introduction}

Long term experience with polymer facings in large hydrodynamic thrust bearings has shown significant improvements in bearing performance. More details on such improvements can be found in [1]. The application of polymers as journal bearing linings is also very promising. Polymer composite linings in hydrodynamic journal bearings provide improved performance during start-up by decreasing the break-away torque [2] and during normal operation by increasing minimum film thickness and extending operating temperature limits. While various polymers can be used, one of the most commonly used materials is polytetrafluoroethylene. A PTFE lined journal bearing is shown in Figure 1.

Since new compliant linings will also affect machine dynamics it is important to quantify their influence on bearing dynamic characteristics. The stability of the bearing is known to be a critical point for some important applications, including turbomachinery. Therefore, any progress in improving the stability is very important.

One common approach used for bearing dynamic analysis is based on a perturbation model (see [3] or [4]). It is based on disturbance of a pressure component and is very efficient for the analysis of rigid babbitted bearings.

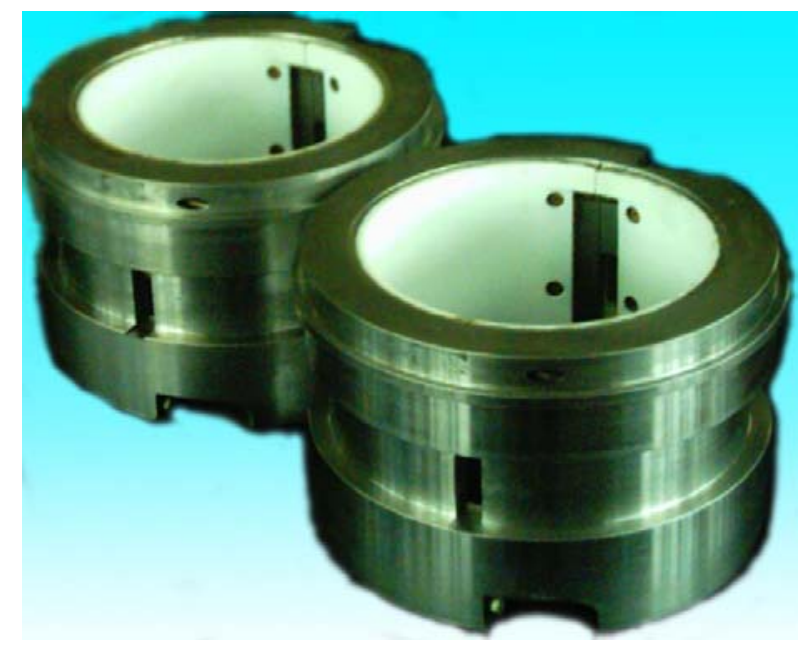

Fig 1 - PTFE lined compliant surface lemon bore journal bearing

In the case of a compliant surface bearing analysis, the disturbance of the pressure implies perturbation of the deformation which is usually neglected as it is believed to be insignificantly small. Several authors (for instance see [5], [6] and [7]) use only the pressure perturbation model to perform dynamic analysis of the bearing.

In this paper we consider and compare two algorithms: one with pressure perturbation only and the other with 
both pressure and deformation perturbations. Our approach is based on Lund's technique [7] and extended to the compliant bearing.

\section{Analysis}

Under the assumption of constant viscosity (isothermal case with relatively low pressure), we can use the following form of the Reynolds equation:

$$
\frac{\partial}{R \partial \theta}\left(\frac{h^{3}}{12 \mu} \frac{\partial p}{R \partial \theta}\right)+\frac{\partial}{\partial z}\left(\frac{h^{3}}{12 \mu} \frac{\partial p}{\partial z}\right)=1 / 2 \Omega \frac{\partial h}{\partial \theta}+\frac{\partial h}{\partial t},
$$

Where $\theta \in[0,2 \pi)$ and $z \in[-L / 2, L / 2]$

The latter forms the mesh grid for the oil film. In the case of a steady-state problem, the simplified form of (1) can be used.

We use the geometry of a lemon bore journal bearing with two supply grooves, each 20 degrees wide, with centres placed at $90-\varphi$ and $270-\varphi$ degrees as shown in Figure 2.

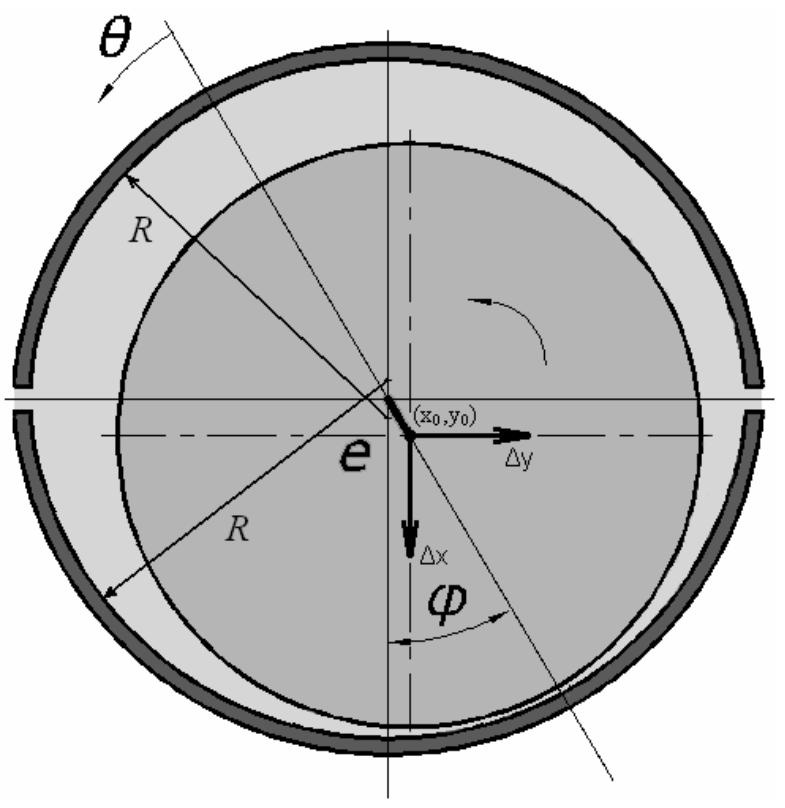

Fig 2 - Bearing geometry.

The displacement of the lobes is set to be a half of the radial clearance which is the difference between $R$ and the shaft radius.

The film thickness is thus given by the formula:

$$
\begin{aligned}
& h_{0}=C \cdot e \cdot \cos \theta-r+ \\
& C\left(\sqrt{\frac{R^{2}}{C^{2}}-\frac{D^{2}}{C^{2}} \sin ^{2}(\theta-\varphi)}-\frac{D}{C}|\cos (\theta-\varphi)|\right.
\end{aligned}
$$

The equation (2) becomes even more complex when we add the perturbance component:

$$
\begin{aligned}
& h=h_{0}+(\Delta x \cos (\theta+\varphi)+\Delta y \sin (\theta+\varphi)), \\
& h_{0}=\left(x_{0} \cos (\theta+\varphi)+y_{0} \sin (\theta+\varphi)\right)-r+ \\
& C\left(\sqrt{\frac{R^{2}}{C^{2}}-\frac{D^{2}}{C^{2}} \sin ^{2}(\theta)}-\frac{D}{C}|\cos (\theta)|\right),
\end{aligned}
$$

where $\left(x_{0}, y_{0}\right)$ is the centre of the shaft (in Cartesian coordinates) and $\Delta x, \Delta y$ are the perturbance components which represent small motions measured from the static equilibrium position towards the vertical and horizontal axes respectively.

The changes in the film thickness lead to the following pressure distribution:

$$
p=p_{0}+p_{x} \Delta x+p_{y} \Delta y+p_{x}^{\prime} \Delta \dot{x}+p_{y}^{\prime} \Delta \dot{y}
$$

where the pressure $p$ is split into different components due to the shaft equilibrium position, shaft displacement and displacement velocities.

Combining (1), (3) and (4) and simplifying the result, one can get a system of five equations:

$$
\frac{\partial}{R \partial \theta}\left(\frac{\left(h_{0}\right)^{3}}{12 \mu} \frac{\partial p_{0}}{R \partial \theta}\right)+\frac{\partial}{\partial z}\left(\frac{\left(h_{0}\right)^{3}}{12 \mu} \frac{\partial p_{0}}{\partial z}\right)=1 / 2 \Omega \frac{\partial h_{0}}{\partial \theta},
$$

$\frac{\partial}{R \partial \theta}\left(\frac{\left(h_{0}\right)^{3}}{12 \mu} \frac{\partial p_{x}}{R \partial \theta}\right)+\frac{\partial}{\partial z}\left(\frac{\left(h_{0}\right)^{3}}{12 \mu} \frac{\partial p_{x}}{\partial z}\right)=-3 \frac{\cos (\theta+\varphi)}{h_{0}} 1 / 2 \Omega \frac{\partial h_{0}}{\partial \theta}$,

$-1 / 2 \Omega \sin (\theta+\varphi)-3\left(\frac{\partial}{R \partial \theta}\left(\frac{\cos (\theta+\varphi)}{h_{0}}\right)\right)\left(\frac{\left(h_{0}\right)^{3}}{12 \mu} \frac{\partial p_{0}}{R \partial \theta}\right)$

$$
\begin{aligned}
& \frac{\partial}{R \partial \theta}\left(\frac{\left(h_{0}\right)^{3}}{12 \mu} \frac{\partial p_{y}}{R \partial \theta}\right)+\frac{\partial}{\partial z}\left(\frac{\left(h_{0}\right)^{3}}{12 \mu} \frac{\partial p_{y}}{\partial z}\right)=-3 \frac{\sin (\theta+\varphi)}{h_{0}} 1 / 2 \Omega \frac{\partial h_{0}}{\partial \theta}, \\
& 1 / 2 \Omega \cos (\theta+\varphi)-3\left(\frac{\partial}{R \partial \theta}\left(\frac{\sin (\theta+\varphi)}{h_{0}}\right)\right)\left(\frac{\left(h_{0}\right)^{3}}{12 \mu} \frac{\partial p_{0}}{R \partial \theta}\right)
\end{aligned}
$$

$$
\frac{\partial}{R \partial \theta}\left(\frac{\left(h_{0}\right)^{3}}{12 \mu} \frac{\partial p_{x}^{\prime}}{R \partial \theta}\right)+\frac{\partial}{\partial z}\left(\frac{\left(h_{0}\right)^{3}}{12 \mu} \frac{\partial p_{x}^{\prime}}{\partial z}\right)=\cos (\theta+\varphi),
$$

$$
\frac{\partial}{R \partial \theta}\left(\frac{\left(h_{0}\right)^{3}}{12 \mu} \frac{\partial p_{y}^{\prime}}{R \partial \theta}\right)+\frac{\partial}{\partial z}\left(\frac{\left(h_{0}\right)^{3}}{12 \mu} \frac{\partial p_{y}^{\prime}}{\partial z}\right)=\sin (\theta+\varphi)
$$

The solution of (6), (7), (8) and (9) gives us stiffness and damping coefficients using the following formulas:

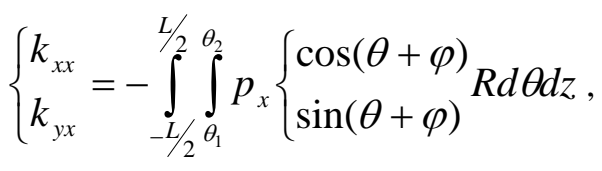




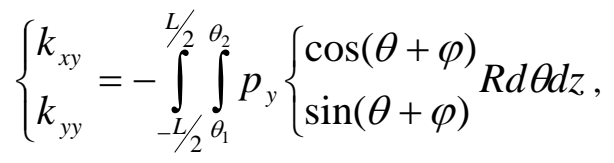

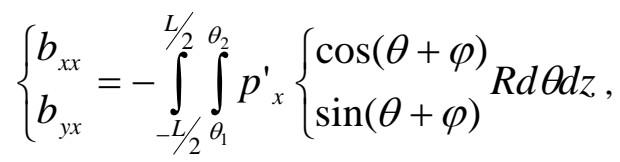

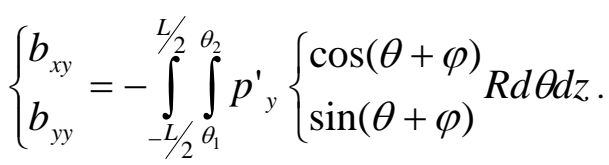

We note that $k_{i j}$ and $b_{i j}$ can be obtained by the derivation of the force components evaluated at the equilibrium position. For instance:

$$
k_{x y}=\left(\frac{\partial F_{x}}{\partial y}\right)_{0} \text { and } b_{x y}=\left(\frac{\partial F_{x}}{\partial \dot{y}}\right)_{0} .
$$

The dimensionless coefficients are determined by

$$
K_{i j}=\frac{C \cdot k_{i j}}{W} \text { and } B_{i j}=\frac{C \cdot 2 \pi \cdot \Omega \cdot b_{i j}}{W}
$$

The set of equations (3)-(13) completes the description of Lund's perturbation method for the rigid babbitted bearing.

\subsection{Simple perturbation model}

Dynamic analysis of the composite lined bearing incorporates the deformation equation:

$$
\delta=\frac{s \cdot p_{0}}{E} \frac{(1+v)(1-2 v)}{1-v} .
$$

We remark that the deformation of the PTFE layer is assumed to be elastic only. The visco-elastic approach needs further investigation and is a subject for future work.

If we let $h^{\prime}=h+\delta$ and resolve equations (5) and (15) simultaneously, we get the solution for a steady-state compliant surface bearing.

Assuming $h_{0}{ }^{\prime}=h_{0}+\delta$ and substituting $h_{0}^{\prime}$ instead of $h_{0}$ into equations (6)-(9), we get $p_{x}, p_{y}, p_{x}^{\prime}$ and $p_{y}^{\prime}$. Finally, applying expressions (10)-(14) we can find $K_{i j}$ and $B_{i j}$.

This approach is well-known and is commonly used for dynamic analysis of compliant surface journal bearings. It is based on the assumption that pressure perturbation is small enough so as to give no influence on the deformation process. Moreover, one can see that the solution is the same as if we were considering the rigid bearing with a profile geometry identical to that of the deformed bearing.

\subsection{Full perturbation model}

To be able to consider the deformation perturbation, we denote

$$
\delta=\frac{s \cdot p}{E} \frac{(1+v)(1-2 v)}{1-v},
$$

where $p$ is defined by (4). Moreover, we put

$$
h=h_{0}+\Delta x \cos (\theta+\varphi)+\Delta y \sin (\theta+\varphi)+\delta .
$$

By substituting (4) and (17) into (1) and simplifying the result, we get the following modifications of equations (5), (7) and (9):

$$
\frac{\partial}{R \partial \theta}\left(\frac{\left(h_{0}+D \cdot p_{0}\right)^{3}}{12 \mu} \frac{\partial p_{0}}{R \partial \theta}\right)+\frac{\partial}{\partial z}\left(\frac{\left(h_{0}+D \cdot p_{0}\right)^{3}}{12 \mu} \frac{\partial p_{0}}{\partial z}\right)=\frac{\Omega}{2} \frac{\partial h_{0}}{\partial \theta}+\frac{\Omega \cdot D}{2} \frac{\partial p_{0}}{\partial \theta},
$$

where

$$
\left.\begin{array}{l}
D=\frac{s}{E} \frac{(1+v)(1-2 v)}{1-v}, \\
\frac{\partial}{R \partial \theta}\left(\frac{\left(h_{0}+D \cdot p_{0}\right)^{3}}{12 \mu} \frac{\partial p_{x}}{R \partial \theta}\right) \\
+\frac{\partial}{\partial z}\left(\frac{\left(h_{0}+D \cdot p_{0}\right)^{3}}{12 \mu} \frac{\partial p_{x}}{\partial z}\right) \\
+\frac{\partial}{R \partial \theta}\left(\frac{\partial p_{0}}{R \partial \theta} \frac{3 D\left(h_{0}+D \cdot p_{0}\right)^{2} \cdot p_{x}}{12 \mu}\right) \\
+\frac{\partial}{\partial z}\left(\frac{\partial p_{0}}{\partial z} \frac{3 D\left(h_{0}+D \cdot p_{0}\right)^{2} \cdot p_{x}}{12 \mu}\right)
\end{array}\right\}=\left\{\begin{array}{l}
\frac{\partial \cdot D}{2} \frac{\partial p_{x}}{\partial \theta}-\frac{\Omega \cdot \sin (\theta+\varphi)}{2}\left(\frac{3\left(h_{0}+D \cdot p_{0}\right)^{2} \cdot \cos (\theta+\varphi)}{12 \mu} \frac{\partial p_{0}}{R \partial \theta}\right), \\
-\frac{\partial}{\partial z}\left(\frac{3\left(h_{0}+D \cdot p_{0}\right)^{2} \cdot \cos (\theta+\varphi)}{12 \mu} \frac{\partial p_{0}}{\partial z}\right)
\end{array}\right.
$$

and

$$
\left.\begin{array}{l}
\frac{\partial}{R \partial \theta}\left(\frac{\left(h_{0}+D \cdot p_{0}\right)^{3}}{12 \mu} \frac{\partial p_{x}^{\prime}}{R \partial \theta}\right) \\
+\frac{\partial}{\partial z}\left(\frac{\left(h_{0}+D \cdot p_{0}\right)^{3}}{12 \mu} \frac{\partial p_{x}^{\prime}}{\partial z}\right) \\
+\frac{\partial}{R \partial \theta}\left(\frac{\partial p_{0}}{R \partial \theta} \frac{3 D\left(h_{0}+D \cdot p_{0}\right)^{2} \cdot p_{x}^{\prime}}{12 \mu}\right) \\
+\frac{\partial}{\partial z}\left(\frac{\partial p_{0}}{\partial z} \frac{3 D\left(h_{0}+D \cdot p_{0}\right)^{2} \cdot p_{x}^{\prime}}{12 \mu}\right)
\end{array}\right\}=\left\{\begin{array}{l}
\frac{\Omega \cdot D}{2} \frac{\partial p_{x}^{\prime}}{\partial \theta} \\
+\cos (\theta+\varphi), \\
+D \cdot p_{x}
\end{array}\right.
$$

The modifications of equations (6) and (8) are quite similar to the ones for (7) and (9).

We remark that the solutions of the modified equations give us a more accurate way to analyse the dynamic characteristics. We also note that we should use the same set of equations (10)-(14) in order to compute the coefficients $K_{i j}$ and $B_{i j}$. 


\subsection{Dynamic characteristics}

As the final step of dynamic analysis, we evaluated coefficients $K_{i j}$ and $B_{i j}$ to compute effective bearing stiffness

$$
k_{0}=\frac{K_{x x} B_{y y}+K_{y y} B_{x x}-K_{x y} B_{y x}-K_{y x} B_{x y}}{B_{x x}+B_{y y}},
$$

square of the instability whirl frequency

$$
\omega_{0}^{2}=\frac{\left(K_{x x}-k_{0}\right)\left(K_{y y}-k_{0}\right)-K_{x y} K_{y x}}{B_{x x} B_{y y}-B_{x y} B_{y x}},
$$

and the critical journal mass (upper level for stability)

$$
M_{\text {crit }}=k_{0} / \omega_{0}^{2} .
$$

\subsection{Geometry and Computational settings}

We use 512x65 (at least 256x33 effective) points for the mesh grid and finite difference approach to discretize the equations. Further on we apply the Jacobi overrelaxation (JOR) technique to get a solution.

We remark that the resolution is chosen to be both fast and precise enough to estimate the dynamic characteristics. The higher resolution gives almost the same result while the computational time increases drastically.

The cavitation effect is handled by implementing a switch-function for the steady-state part of the problem (i.e. equation (5) and its modifications) and by determining specific boundary conditions for the rest of the equations. The level of cavitation is set to be zero (i.e. ambient pressure).

We assume ambient pressure at the bearing sides and within the supply groove areas.

Bearing parameters used in the computations are as follows: bearing ratio $L / D=0.5$ and $L / D=1$, the relative radial clearance $\mathrm{Cr} / \mathrm{R}=0.0016$ and displacement ratio $\delta / C r=1 / 2$. The soft layer is made of PTFE and its thickness varies from $5 \mathrm{Cr}$ to $25 \mathrm{Cr}$. We set Young's modulus and Poisson's ratio to be $0.11 \mathrm{GPa}$ and 0.46 respectively. The fluid film viscosity is assumed to be constant and equal to $63 \mathrm{mPa} \cdot \mathrm{s}$.

\section{Results}

We start with verification of our algorithms to the reference data computed by Lund [6]. Figure 3 shows dimensionless Sommerfeld number defined by

$$
S=\frac{\eta \omega L R}{\pi W}\left(\frac{R}{C}\right)^{2}=\frac{1}{\pi \bar{W}}
$$

where $W$ and $\bar{W}$ are normal and dimensionless loads respectively.

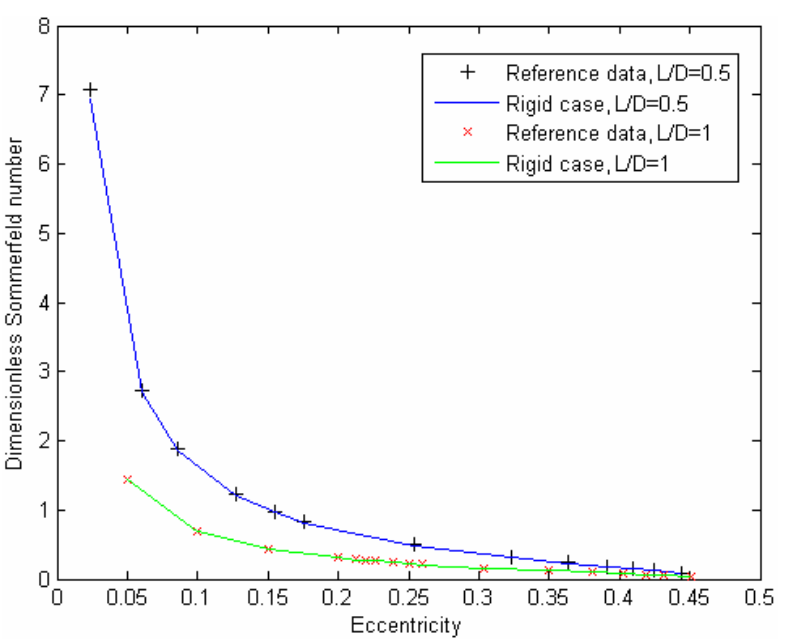

Fig 3 - Sommerfeld number.

We can see good agreement for both bearing ratios, i.e. short when $L / D=0.5$ and long when $L / D=1$.

The attitude angle as well as bearing stiffness and damping coefficients almost coincide with those provided in Lund's paper, see for example Figure 4.

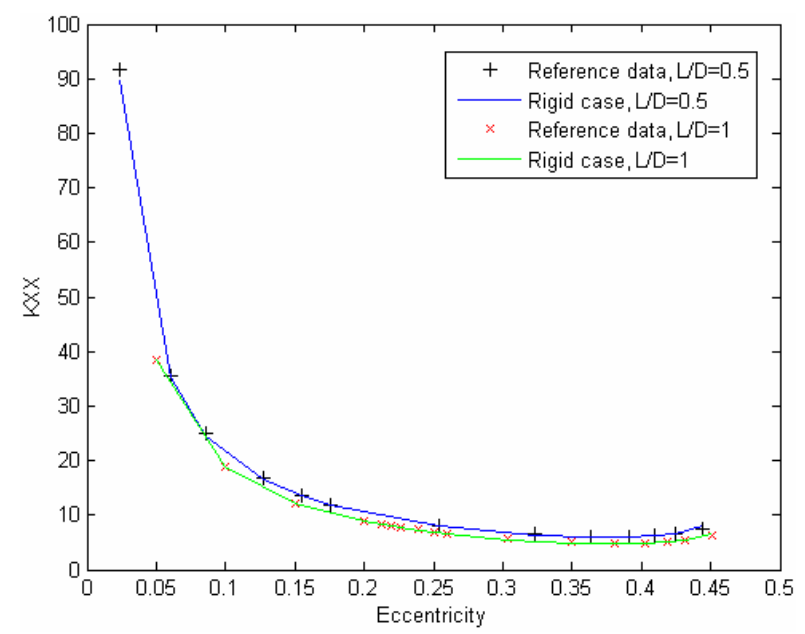

Fig 4 - Kxx coefficient.

Since the verification appears to be sufficient, we proceed to the main results, showing our expectations of the PTFE layer performance. Due to similarities between trends for different ratios we present more detailed results for the short bearing only (Figures 5-14 and 17) and give examples of those for the long one (Figures 15-16 and 18).

Figure 5 and 6 show the Sommerfeld number and the attitude angle as functions of eccentricity. We remind that the bearing ratio is fixed to $L / D=0.5$ and soft layer thickness varies from $5 \mathrm{Cr}$ to $25 \mathrm{Cr}$. 


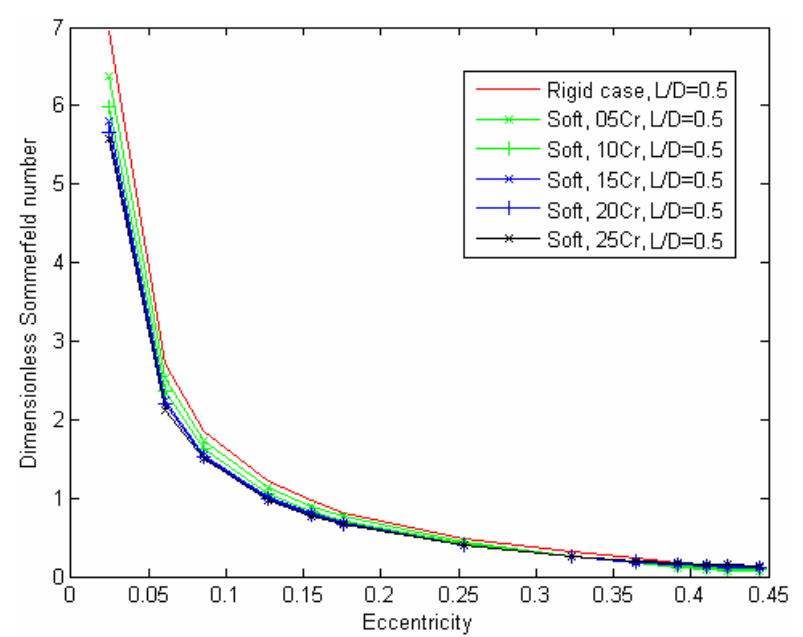

Fig 5 - Sommerfeld number.

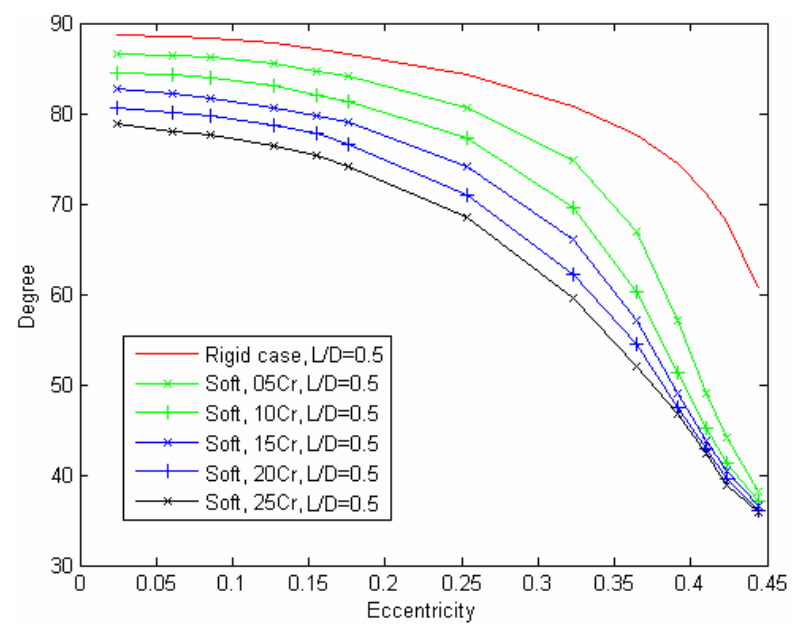

Fig 6 - attitude angle.

Figure 5 shows very low sensitivity of the Sommerfeld number to the PTFE layer thickness. By contrast, attitude angle decreases significantly with the increasing thickness of the soft layer (see Figure 6). This decrease is especially pronounced at higher eccentricities.

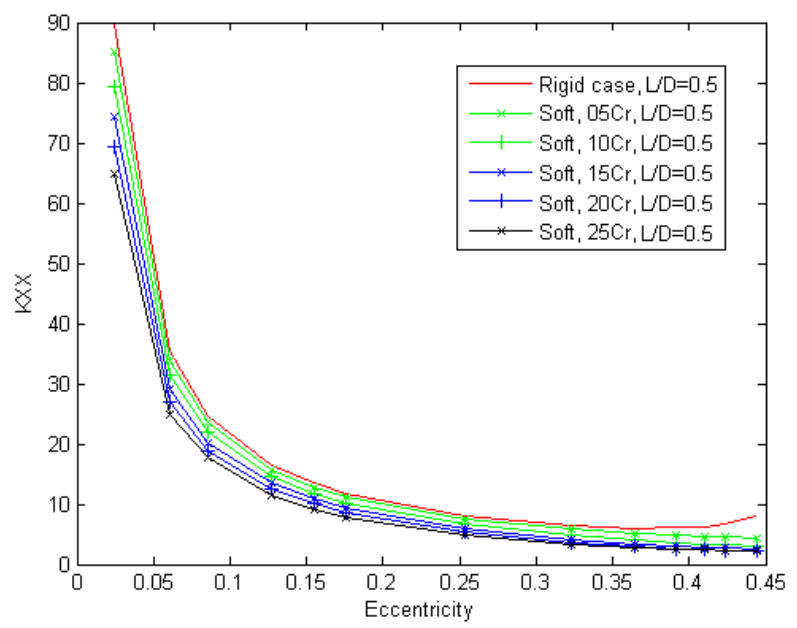

Fig 7 - Kxx coefficient.

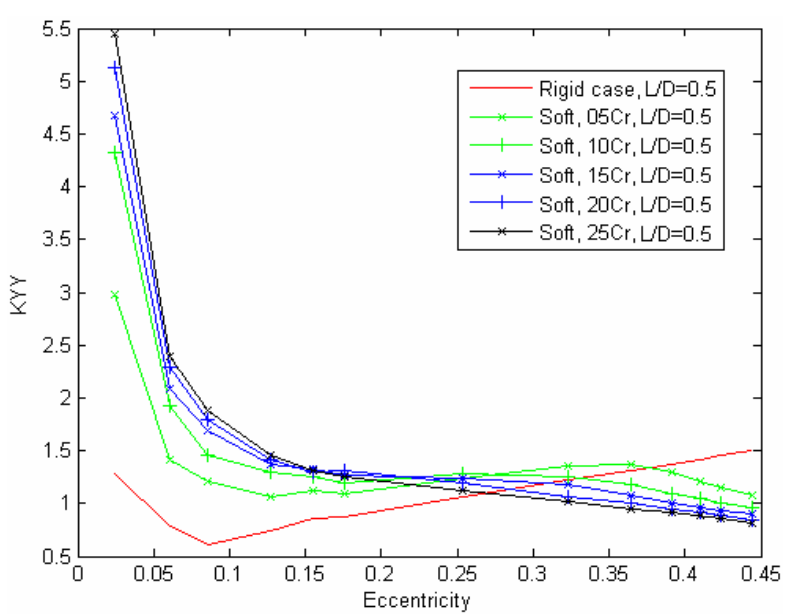

Fig 8 - Kyy coefficient.

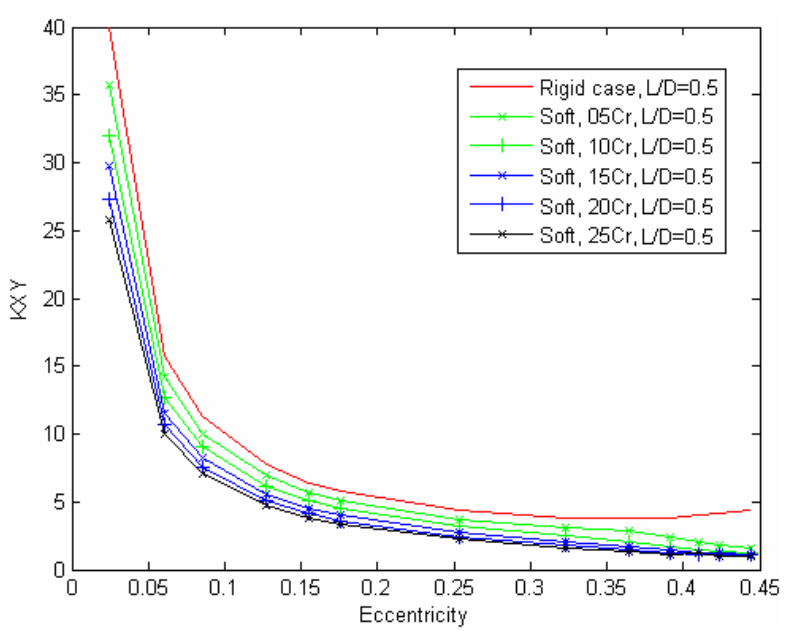

Fig 9 - Kxy coefficient.

Figures 7-10 show stiffness coefficients as a function of eccentricity. Kxx and Kxy (Figures 7 and 9) are lower for the soft bearing at all eccentricities. Kyy and Kyx (Figures 8 and 10) increase with an increase in layer thickness at low eccentricities, but Kyy becomes lower at high eccentricities.

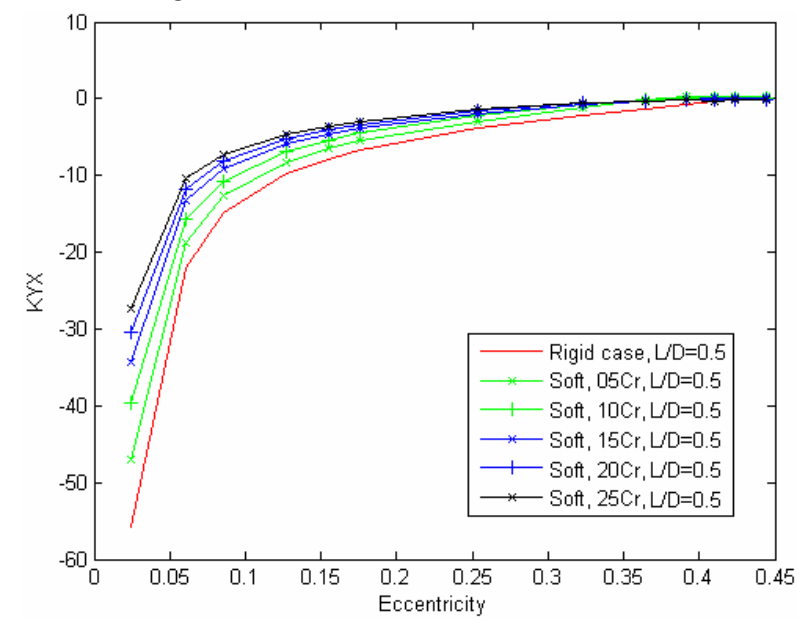

Fig 10 - Kyx coefficient. 
Four damping coefficients as a function of eccentricity are shown in Figures 11-14.

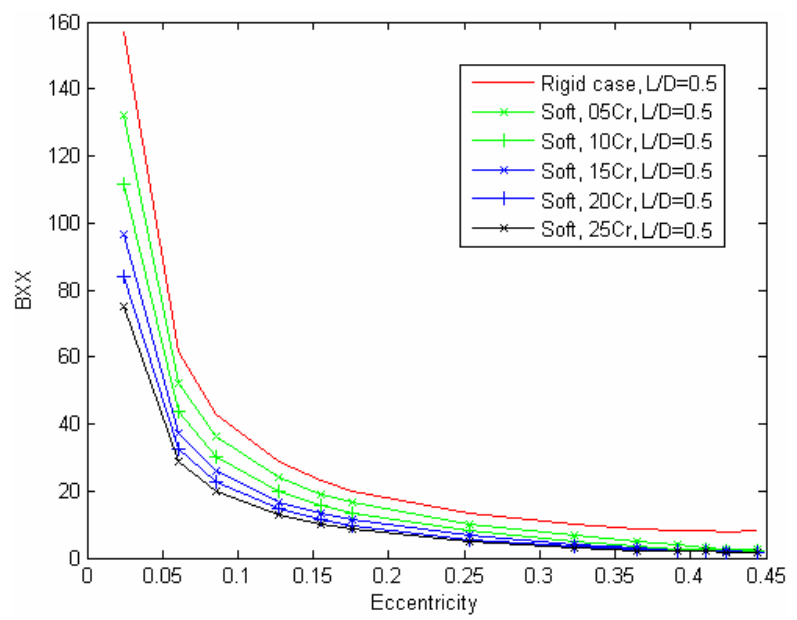

Fig 11 - Bxx coefficient.

Increasing thickness of the soft layer leads to a decrease in both Bxx and Byy damping coefficients (Figures 11 and 12).

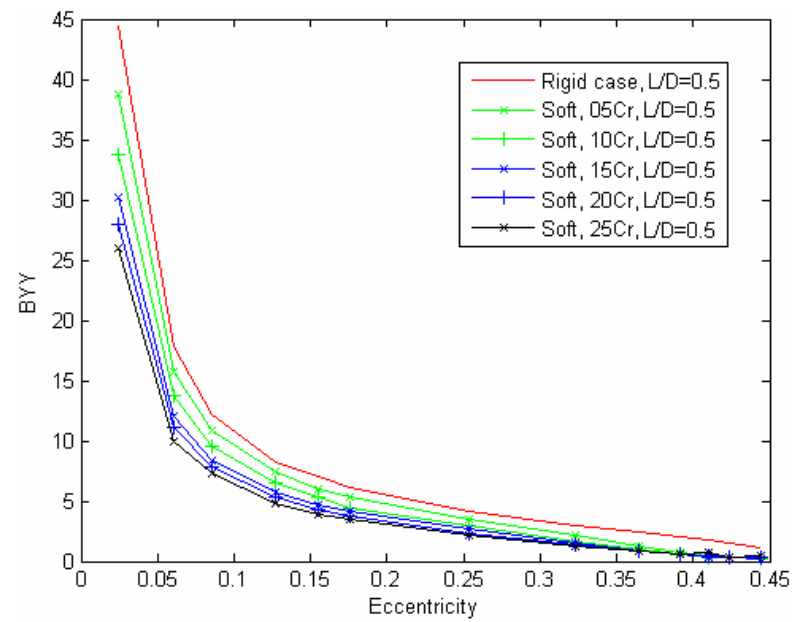

Fig 12 - Byy coefficient.

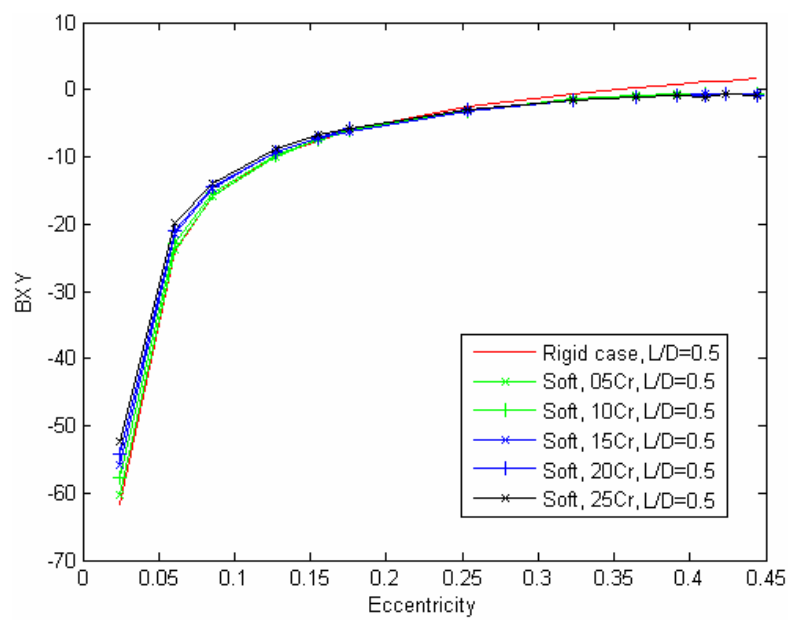

Fig 13 - Bxy coefficient.
Bxy (Figures 13) is only slightly affected by the elasticity of the bearing lining.

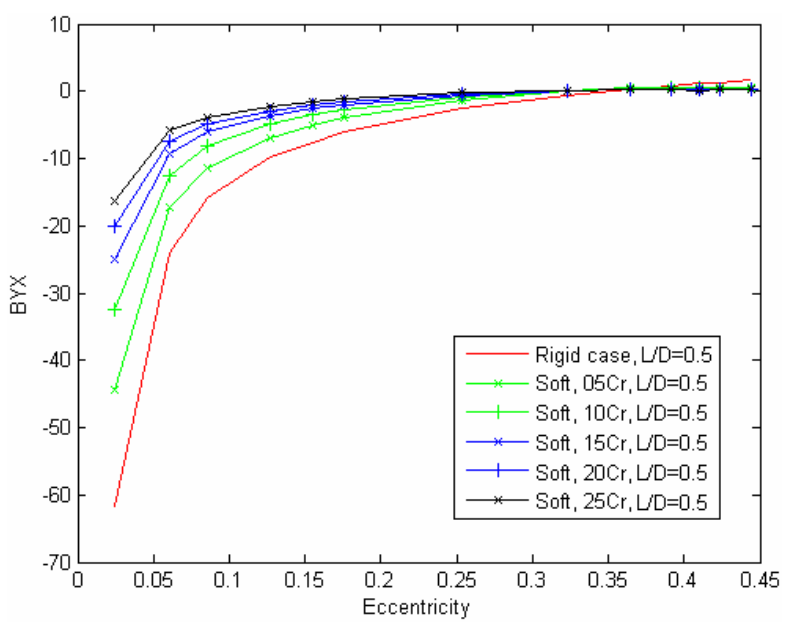

Fig 14 - Byx coefficient.

Byx (Figure 14) is strongly affected by the bearing elasticity while its behaviour is opposite for low and high eccentricities.

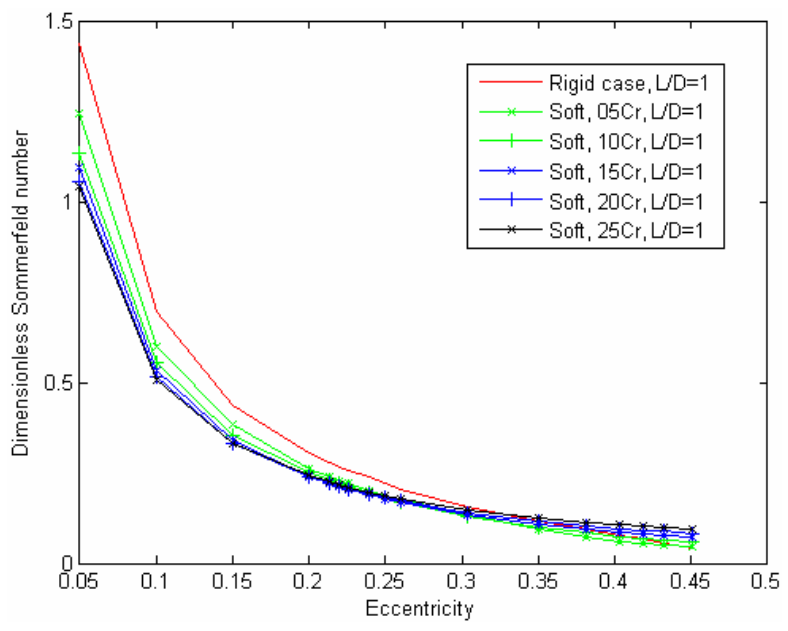

Fig 15 - Sommerfeld number.

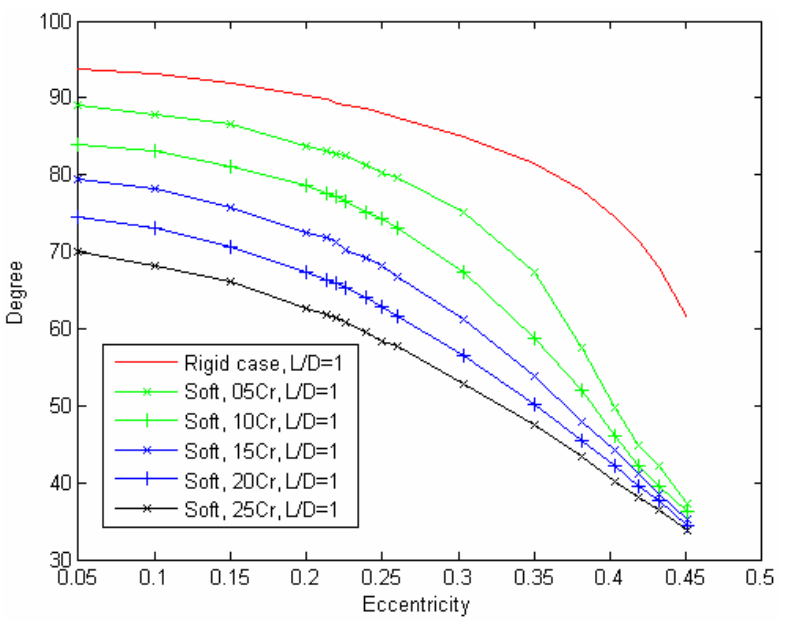

Fig 16 - Attitude angle. 


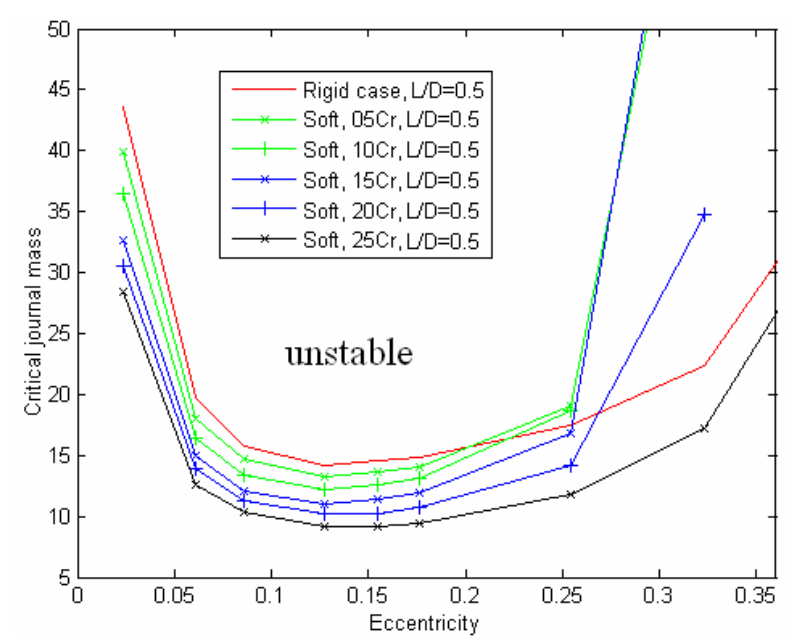

Fig 17 - Critical journal mass, short bearing.

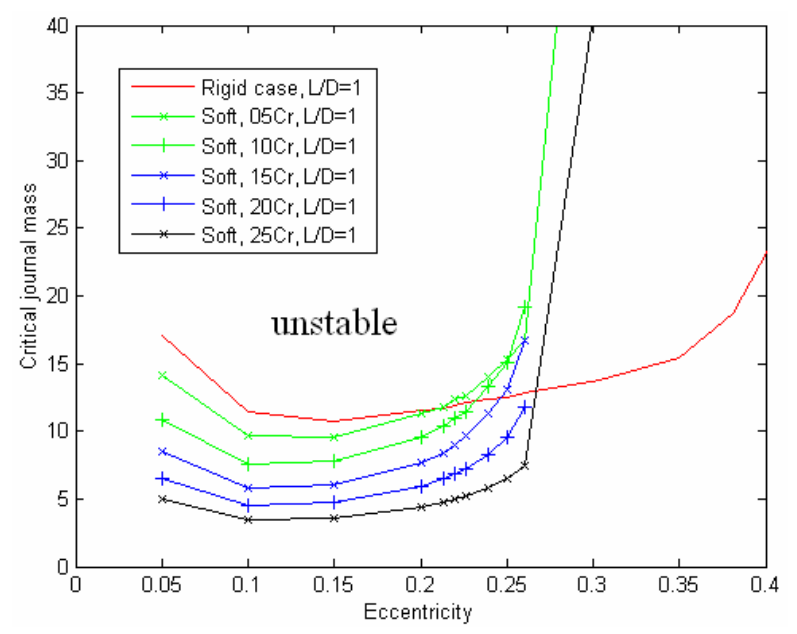

Fig 18 - Critical journal mass, long bearing.

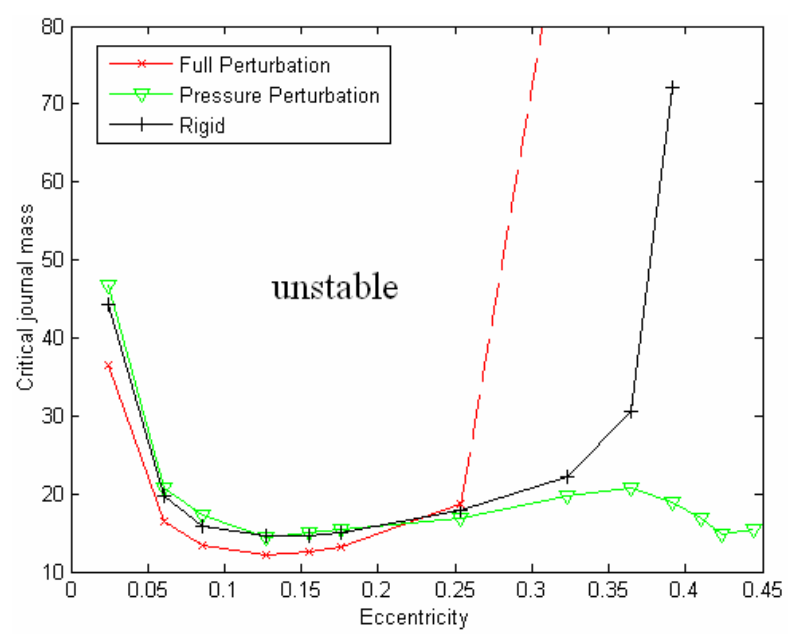

Fig 19 - Critical journal mass.

Similar trends in bearing characteristics can be observed for a wider bearing ( $L / D=1$ ). Sommerfeld number and attitude angle are presented in Figures 15 and 16, while bearing stiffness and damping are omit- ted due to the paper length limitation. Similar influence of bearing elasticity on Sommerfeld number and attitude angle can be seen by comparison of Figures 5 and 6 with 15 and 16.

Figures 17 to 18 represent dimensionless critical journal mass. As can be observed, compliant bearing stability is reduced for eccentricities below 0.21-0.27. This reduction depends on the layer thickness or the magnitude of the layer deformation. Elastic deformation of the bearing liner improves stability at higher eccentricities. At the same time, an excessive layer deformation is detrimental as can be seen for the short bearing with the thickest PTFE layer (Figure 17). A similar effect can be observed for the wider bearing if the PTFE layer thickness is further increased.

Figure 19 shows dimensionless critical journal mass computed for the rigid and soft bearings. It can be seen that the pressure perturbation approach shows a decrease in stability at high eccentricities whereas full perturbation approach shows an improvement in stability.

\section{Discussion}

The effect of the PTFE layer on bearing stability varies depending on the eccentricity and liner thickness. In the low eccentricity range, a decrease in stability can be observed. The deformation changes the bearing geometry making it closer to the cylindrical one and decreasing the effect of preload. As a result we observe a decrease in bearing stability compared to the rigid case.

In contrast to these results, improvement of stability can be seen for higher eccentricities. However, this effect strongly depends on the soft layer thickness and even can be unreachable for certain parameters (see Figures 17 and 18). Therefore, the properties of the compliant layer are of great importance and should be chosen according to the bearing geometry and desired eccentricity range.

The influence of bearing geometry (width) is not as clear. The short bearing seems to be affected more by the soft liner implementation than the long one. Moreover, Figures 17 and 18 show the higher sensitivity of the short bearing to the soft layer thickness.

Since the EHD model does not consider side leakage effects, the situation might change for the TEHD case. This is a question for further investigation in the future. Finally we remark that the simple approach shows completely different results to those found from the full perturbation mode when applied to the compliant lined bearing (see Figure 19). It is clear that the perturbation of the deformable layer is as important to consider as the perturbation of the pressure itself. Moreover it is not difficult to see that the classical approach can not distinguish the PTFE lined bearing from the babbitted bearing with a specific geometry profile, for any fixed eccentricity (though the profile is different 
for different eccentricities). Therefore, the extended approach, presented in this paper, should be used for the lined bearings whenever the deformation is not negligible.

\section{Conclusions}

A comparative dynamic analysis of a conventional babbitted lemon bore bearing and a compliant lemon bore bearing shows that:

1. Load carrying capacity of the compliant bearing is increased at low eccentricities and reduced at high eccentricities.

2. Attitude angle of the compliant bearing is reduced.

3. Cross couple damping coefficients for a compliant bearing are not equal.

4. Stability of the compliant bearing can be significantly improved at high eccentricities by a proper choice of the layer compliancy.

\section{Acknowledgement}

The financial support provided by the Swedish Energy Agency, ABB Automation Technologies, Alstom Hydro, Elforsk, Evonik RohMax Additives GmbH, Statoil Lubricants and Siemens Industrial Turbomachinery is gratefully acknowledged.

\section{Nomenclature}

$\theta=$ circumferential coordinate

$Z$ = axial coordinate

$\mu=$ reference viscosity.

$\mathrm{Cr}=\mathrm{R}-r$ is the radial clearance

$R=$ bearing radii

$D=$ diameter of the bearing

$r=$ shaft radii

$\delta=$ displacement of the lobes

$h=$ fluid film thickness

$p=$ pressure distribution

$p_{0}=$ steady-state pressure distribution

$p_{x}, p_{y}=$ components of the pressure connected to the displacement of the shaft in $x$ and $y$ directions.

$p^{\prime}{ }_{x}, p^{\prime}{ }_{y}=$ component of the pressure connected to the velocity of the displacement in $x$ and $y$ directions.

$F_{x}, F_{y}=$ force components in $x$ and $y$ directions.

$e=$ eccentricity

$\varphi=$ attitude angle

$\Omega=$ rotation speed, rpm

$\delta=$ deformation of the soft layer

$S=$ soft layer thickness

$E=$ Young's modulus $=0.11 \mathrm{GPa}$

$v=$ Poisson's ratio $=0.46$
$W=$ load

$\bar{W}=$ dimensionless load

$S=$ Sommerfeld number

$k_{i j}=$ stiffness coefficient

$K_{i j}=$ dimensionless stiffness coefficient (see 14)

$b_{i j}=$ damping coefficient

$B_{i j}=$ dimensionless damping coefficient (see 14)

$k_{0}=$ effective bearing stiffness (see 18)

$\omega_{0}=$ whirl frequency (see 19)

$M_{\text {crit }}=$ critical journal mass (see 20)

\section{Reference}

[1] S.Glavatskih, "Tilting pad thrust bearings," Proc. $29^{\text {th }}$ Leeds-Lyon Symposium on Tribology, Leeds, 2002. p. 379- 390.

[2] McCarthy D.M.C. and Glavatskih S.B. “'BreakAway' Friction for PTFE-Based Composites”, $16^{\text {th }}$ International Colloquium Tribology, Esslingen, Jan 2008.

[3] Klit P. and Lund J.W. "Calculation of the Dynamic Coefficients of a Journal Bearing Using a Variational Approach", Journal of Tribology, Transactions of the ASME, 108, n 3, Jul, 1986, p 421-425.

[4] Lund J.W., "Review of the Concept of Dynamic Coefficients for Fluid Film Journal Bearings“, Journal of Tribology, Transactions of the ASME, 109, n 1, Jan, 1987, p 37-41.

[5] Lund J.W. and Thomsen J.W. “A Calculation Method and Data for the Dynamic Coefficients of Oil-Lubricated Journal Bearings", Bulletin of the International Association of Engineering Geology, 1978, p 1-28.

[6] Rao T.V.V.L.N. and Sawicki J.T. "Dynamic coefficient prediction in multi-lobe journal bearings using a mass conservation algorithm”, Trib Trans., 46, n 3, 2003, p 414-420.

[7] Prabhakaran Nair, Sinhasan K and Singh DV "Elastohydrodynamic effects in elliptical bearing”, Wear ,118, 1987, p 129-145.

[8] Prabhakaran Nair, K., Sukumaran Nair, V.P. and Jayadas N.H. "Static and Dynamic Analysis of Elastohydrodynamic Elliptical Journal Bearing with Micropolar Lubricant“, Tribology International, 40, n 2, 2007, p 297-305.

[9] Ramesh J., Majumdar B.C. and Rao N.S. "Stability characteristics of rough submerged oil elliptical bearings under dynamic load“, Tribology International, v 30, n 12, 1997, p 857-863. 